\title{
Clinical analysis of preoperative risk factors for the incidence of deep venous thromboembolism in patients undergoing posterior lumbar interbody fusion
}

\author{
Jingchao Wei $i^{3}$ Wenyi $\mathrm{Li}^{3}$, Yueying Pei ${ }^{3,4}$, Yong Shen ${ }^{1,2}$ and Jia $\mathrm{Li}^{1,2^{*}}$
}

\begin{abstract}
Background: The purpose of this study aimed to assess preoperative risk factors for the incidence of deep venous thromboembolism in patients undergoing posterior lumbar interbody fusion (PLIF).

Methods: The diagnosis of preoperative deep vein thrombosis (DVT) was confirmed by Doppler ultrasonography. To examine the preoperative risk factors for DVT admitted for PLIF, comparative analysis of the DVT-positive and DVT-negative groups was done.

Results: DVT was detected in $9.4 \%$ (269/2861) patients, including 17 proximal DVT patients (6.3\%) and 252 the distal DVT patients (93.7\%). According to multivariate logistic regression analysis, the age, preoperative D-dimer, and history of rheumatoid arthritis were significant risk factors relative to the onset of DVT after posterior lumbar surgery.
\end{abstract}

Conclusions: According to the result of our study, age, positive preoperative plasma D-dimer level, and rheumatoid arthritis had the influential impact on the incidence of DVT admitted for PLIF.

Keywords: Deep venous thrombosis, Posterior lumbar interbody fusion, Preoperative risk factors, Preoperative plasma D-dimer level

\section{Background}

Venous thromboembolism (VTE) is a common complication of spinal surgery which included deep vein thrombosis (DVT) and pulmonary embolism (PE). Many studies had reported the risk factors for DVT development in patients who undergo spine surgery, such as advanced age, obesity, neurological deficit, hospitalization, major surgery, immobilization, blood transfusion, malignancy, and trauma. Risk factors of DVT were common in patients with degenerative spine diseases [1-3]. Without prophylaxis, approximately $15 \%$ of patients undergoing posterior spinal surgery develop DVT [4].

\footnotetext{
*Correspondence: ljlyqbwin2010@yeah.net

'Department of Orthopaedic Surgery, The Third Hospital of Hebei Medical University, 139 Ziqiang Road, Shijiazhuang 050051, People's Republic of China

${ }^{2}$ The Key Laboratory of Orthopedic Biomechanics of Hebei Province, The Third Hospital of Hebei Medical University, Shijiazhuang 050051, People's Republic of China

Full list of author information is available at the end of the article
}

The need for appropriate prophylaxis was increasingly accepted. There were variety of strategies, including mechanical and chemical prophylaxis, which had been shown to be effective in preventing postoperative VTE [5-7]. The low molecular weight heparin (LMWH) had been used as chemical prophylaxis in decreasing the incidence of VTE in patients after spinal surgery. However, there was still got the fear of hemorrhagic disorders and spinal epidural hematoma (SEH) [8]. Therefore, it was still a matter of debate about the choice of prophylaxis in patients following spinal surgeries regardless of these strategies were used to prevent VTE.

D-dimer was widely used to diagnose VTE and markedly high in comparison to those in healthy volunteer [9]. However, there was insufficient published data focus on whether patients with preoperative plasma D-dimer level needed to use preoperative chemical prophylaxis. Furthermore, the efficacy of using preoperative LMWH on incidence of VTE or hemorrhagic disorders and SEH after 
surgery was still unknown. The purpose of this study aimed to assess the effect of preoperative plasma Ddimer level on the incidence of deep venous thromboembolism in patients undergoing posterior lumbar interbody fusion (PLIF).

\section{Methods}

This study retrospectively reviewed of patients who underwent posterior lumbar interbody fusion, in the authors' center between March 2008 and November 2014. Patients were diagnosed with lumbar pathologies such as lumbar disc herniation, lumbar spinal stenosis, and isthmic spondylolisthesis, by clinical and physical examinations corresponded to manifestations of lumbar spine radiographs, computed tomography (CT) scans, and magnetic resonance images (MRI). Patients who had preoperative urinalysis positive for red blood cells, fecal occult blood, skin purpura, or hematoma; fracture, infection, or tumor; active bleeding or high risk of bleeding; oral anticoagulant therapy 1 week prior to the surgery; and LMWH hypersensitivity were excluded from this study. Those patients with a previous history of DVT or the existence of DVT was detected preoperatively were also excluded from this study. The preoperative plasma D-dimer level was defined before the study as a plasma concentration of $0.50 \mu \mathrm{g} / \mathrm{ml}$ or more, as recommended by the manufacturer.

All patients received mechanical prophylaxis (intermittent pneumatic compression devices) from induction of general anesthesia to postoperative ambulation. The patients were routinely administered daily LMWH (Enoxaparin, $40 \mathrm{mg} /$ day) from postoperative day (POD) 1 to POD 7. For the patients with preoperative positive value of $\mathrm{D}$-dimer, the first subcutaneous injection was given $12 \mathrm{~h}$ prior to surgery. Walking exercise started 5 days after surgery.

Both groups had a daily physical evaluation in order to find early onset DVT symptoms and symptomatic epidural/wound hematoma during hospitalization. Doppler ultrasonography was performed on patients within $24 \mathrm{~h}$ of admission to the hospital, and the second examination were performed 5 days postoperatively. In cases where DVT symptoms (warmth, erythema, unilateral calf pain, edema) were detected after the operation, an additional Doppler ultrasonography was performed on the patient.

This study was approved by the Institutional Review Board of the Third Hospital of Hebei Medical University. Subjects provided informed consent to participate in the study; those who did not wish to participate in this study were not enrolled.

\section{Statistical analysis}

Statistical analysis was performed with SPSS 17.0 for Windows (SPSS Inc., Chicago, IL, USA). The patients' characteristics between groups were compared using the
Mann-Whitney $U$ test and Fisher's exact test. After these univariate analyses, a $P<0.2$ was selected and evaluated by multivariate logistic regression analysis to identify predictors of DVT among independent variable. Conclusively, a $P<0.05$ was considered statistically significant.

\section{Results}

In this retrospective study, 3629 patients initially identified, a total of 2861 patients were admitted into analyses, and 768 were out for adequate clinical information in the medical record. According to the incidence of DVT, 269 patients were classified into DVT-positive group and 2592 patients comprised DVT-negative group. In this series, patients completed the study without any lifethreatening complications. There were no patients with clinical signs of DVT or PE. However, 6 cases $(0.21 \%)$ developed SEH in 2 days after surgery.

Of 269 DVT-positive patients, the distal thrombus was identified in 252 (93.7\%); the proximal thrombus only was identified in 17 (6.3\%). The incidence of DVT was significantly higher in the preoperative $\mathrm{D}$-dimer-positive patients compared to the $\mathrm{D}$-dimer-negative patients $(P=0.000)$. The mean age in DVT-positive group was significantly older than that in DVT-negative patients $(P=0.002)$.

The most frequent preoperative medical history in DVTpositive group was hypertension (137 patients, $50.9 \%$ ); the second was coronary heart disease (102 patients, $37.9 \%$ ). Rheumatoid arthritis was seen in $0.5 \%(12 / 2592)$ of the DVT-negative group and $11.5 \%(31 / 269)$ of the DVTpositive group $(P<0.05)$. There were $32.0 \%(82 / 269$ patients) of the DVT-positive group who had undergone major surgery, but only $25.0 \%(648 / 2592)$ of the DVTnegative group had undergone major surgery $(P=0.006)$ (Table 1).

Table 1 Univariate analysis of the risk factors for DVT

\begin{tabular}{llll}
\hline Characteristic & DVT (+) group & DVT $(-)$ group & $P$ value \\
\hline No. of patients & 269 & 2595 & \\
Age & $61.3 \pm 10.3$ & $52.6 \pm 11.7$ & 0.002 \\
Gender(M/F) & $129 / 140$ & $1382 / 1213$ & 0.097 \\
BMI $\left(\mathrm{kg} / \mathrm{m}^{2}\right)$ & $24.2 \pm 3.6$ & $23.5 \pm 2.9$ & 0.376 \\
Hypertension & 137 & 1509 & 0.066 \\
Coronary heart disease & 102 & 956 & 0.301 \\
Diabetes mellitus & 89 & 823 & 0.633 \\
Rheumatoid arthritis & 31 & 12 & 0.000 \\
Major surgery & 82 & 648 & 0.164 \\
Lumbar pathologies & & & 0.659 \\
Lumbar disc herniation & 87 & 901 & \\
Lumbar spinal stenosis & 107 & 1026 & \\
Isthmic spondylolisthesis & 75 & 668 & 0.000 \\
\hline Preoperative D-dimer $( \pm)$ & $112 / 157$ & $89 / 2056$ & \\
\hline
\end{tabular}


To compare the relative impact of these variables on the incidence of DVT, multiple logistic regression analysis was performed. Statistically significant risk factors for DVT included preoperative plasma D-dimer level, age, hypertension, rheumatoid arthritis, and major surgery in the univariate analysis. The multivariate analysis showed that preoperative plasma D-dimer level, age, and rheumatoid arthritis were significant independent risk factors for DVT admitted PLIF (Table 2).

\section{Discussion}

As we all know, PLIF was likely to be associated with postoperative DVT in spinal surgery. The findings of our study indicated that although demographics and basic characteristics associated with DVT-positive and DVTnegative group were generally similar, DVT-positive was associated with a significantly high preoperative plasma D-dimer level compared to the DVT-negative group. According to the chronic disease history, patients with rheumatoid arthritis were significantly high incidence in DVT group. It also provided evidence for the safety of LMWH at preoperative and postoperative in PLIF. The multivariate analysis showed that preoperative plasma D-dimer level, age $(P<0.013)$, and rheumatoid arthritis $(P=0.028)$ were significant independent preoperative risk factors for DVT admitted for PLIF.

D-dimer testing had been widely used in the exclusion of DVT/PE. A positive D-dimer test usually indicates blood clots or thrombosis in the body, which is associated with the severity of DVT. A standard cutoff of $0.50 \mu \mathrm{g} / \mathrm{ml}$ was used in routine clinical practice, with values above that level considered positive. However, the customary D-dimer cutoff is inappropriate under different conditions, including infection and pregnancy. Previous studies had shown that D-dimer level of patients undergoing surgical procedures would result from the development of DVT [9]. D-dimer level was a negative predictor for DVT which was helpful for diagnostic aid in suspected DVT, however, with a not very high sensitivity as it had been reported [10]. Whether an elevated level of D-dimer before PLIF was associated with the occurrence of postoperative DVT has not been definitely established and warrants further investigation. In this study, the incidence of DVT was significantly higher in

Table 2 Multivariate analysis of the risk factors for DVT

\begin{tabular}{llll}
\hline Risk factors & $P$ & OR & $95 \% \mathrm{Cl}$ \\
\hline Age & 0.021 & 1.0261 & $0.9810-1.0643$ \\
Gender & 0.298 & 0.1833 & $0.0043-5.2853$ \\
Hypertension & 0.156 & 1.5422 & $0.9853-0.9368$ \\
Preoperative D-dimer & 0.013 & 6.2217 & $1.0354-2.9310$ \\
Major surgery & 0.113 & 0.1512 & $0.0694-0.9393$ \\
Rheumatoid arthritis & 0.003 & 6.1203 & $1.0412-42.9899$ \\
\hline
\end{tabular}

the preoperative $\mathrm{D}$-dimer positive patients compared to the negative patients $(P<0.05)$. The multivariate analysis showed the preoperative plasma D-dimer level was significant independent preoperative risk factors for DVT admitted for PLIF.

Reducing the risk of VTE in patients undergoing spinal surgery, it had recommended combined mechanical and chemical prophylaxis. Oda et al. had found the prevalence of DVT after posterior spinal surgery without prophylaxis was $15.5 \%$ which was higher than generally recognized [4].

The type of common prophylactic treatment after spinal surgery had been used including mechanical (intermittent pneumatic compression) and chemical prophylaxis (LMWH). The use of LMWH was the most effective methods, which was preferred by most surgeons $[8,11,12]$. However, there was still controversy over the effect of LMWH prophylaxis in spinal surgery because of the fear of SEH and bleeding complications. Glotzbecker et al. had compared the effect of mechanical prophylaxis with chemical prophylaxis after spinal surgery. It had suggested that the use of mechanical prophylaxis could be a primary method to decrease the incidence of DVT. Furthermore, the chemical prophylaxis was associated with the lowest prevalence of DVT incidence after spinal surgery and no evidence to support or refute the use of chemical anticoagulants in routine elective spinal surgery [13]. Strom et al. retrospectively reviewed cases of cervical and lumbar laminectomy and found low hemorrhage risk with LMWH prophylaxis after spinal surgery. In their opinion, patients undergoing laminectomy for degenerative disease without LMWH prophylaxis might be at significant risk for DVT[7]. Gerlach et al. reported the results of 1299 patients who underwent lumbar surgery. To prevent DVT, all patients were routinely treated subcutaneously with mechanical and chemical prophylaxis until discharge. Their results confirmed that early postoperative chemical prophylaxis in patients with lumbar surgery without an increased risk of postoperative hemorrhage [14]. This study assessed the effect of LMWH on positive preoperative plasma D-dimer level patients undergoing PLIF. From this study, we could conclude that probably, medication of LMWH as a prophylaxis has not adversely significant hemorrhagic or SEH. For preoperative positive value of D-dimer patients, combinations of mechanical and chemical prophylaxis were strongly recommended.

Although statistical support for spine-specific risk factors was lacking, several studies had noted common observations for patients who sustained a DVT. Previous studies had demonstrated the risk factors associated with DVT after spinal surgery, including advanced age, Ddimer, previous thromboembolism, ratio of perioperative immobilization, type of spinal surgery, and hypertension. Sansone et al. analyzed a total of 4383 patients who had 
undergone elective spine surgery. In their study, the risk of DVT was relatively low, especially for the patients who receive chemical prophylaxis. Unfortunately, chemical prophylaxis exposes patients to a greater risk of hemorrhagic disorders and SHE [15]. Yoshioka et al. reported the statistically significant risk factors for DVT including advanced age, neurologic deficits, and spinal tumor. Of these factors, spinal tumor was a high risk of DVT. In another study, the fibrin monomer complex measured 1 day after surgery was considered to be useful as an indicator of DVT $[16,17]$. Oda et al. found a statistically significant risk of VTE associated with advanced age and anatomic location of surgery [4].

In the present study, the results indicated that more patients with a positive preoperative plasma D-dimer level in DVT group than non-DVT group. However, there was no significant difference among the lumbar pathologies. Therefore, it is believed that the lumbar pathology itself had not a higher impact on the incidence of VTE. Univariate analysis found that positive preoperative plasma D-dimer level, advanced age, history of rheumatoid arthritis, hypertension, and major surgery tended to be risk factors for DVT. According to the multivariate logistic regression analysis, it had shown that positive preoperative plasma D-dimer level, advanced age, and history of RA were independent risk factors for postoperative DVT in patients admitted for PLIF.

Previous studies have found elevated risks of DVT in patients with rheumatoid arthritis undergoing joint arthroplasty [18-20]. It had been confirmed that patients with rheumatoid arthritis had an increased risk of VTE compared to the general population. According to this study, the prevalence of postoperative DVT was significantly higher in rheumatoid arthritis patients admitted for PLIF than others. Patients with rheumatoid arthritis got an increased risk of developing DVT, which might be attributable to the presence of systemic inflammatory disease. Moreover, the use of corticosteroids might accelerate atherosclerotic disease.

\section{Limitation}

However, there were several limitations in this study. Owing to insufficient information, this study could not possible to give a suggestion on standardized preoperative chemical prophylaxis protocol. Whether mechanical prophylaxis alone or combined with chemical prophylaxis could be efficacious on the positive preoperative plasma D-dimer level is unknown. Further studies are warranted to investigate preoperative plus postoperative prophylaxis for patients undergoing other spinal surgeries. Furthermore, it must be investigated the necessity of the use of LMWH prophylaxis, its initiation time and length, particularly in high-risk patients. A prospective multiplecenter study are needed to assess the safety of early LMWH administration which would certainly provide more useful information. In the future, we will further investigate the relationship between RA associated D-dimer increase and DVT.

\section{Conclusions}

According to the result of our study, age, positive preoperative plasma D-dimer level, and rheumatoid arthritis had the influential impact on the incidence of DVT admitted for PLIF. For positive preoperative plasma D-dimer level patients, combinations of mechanical and chemical prophylaxis were recommended. Surgeons should be pay attention to decrease the incidence of DVT if specific preoperative risk factors were present.

\section{Acknowledgements}

We thank Dr. Yingze Zhang for his support in obtaining the approval of the ethics committee for this study.

\section{Authors' contributions}

$J \mathrm{~L}$ carried out the conception and design of the study and acquisition and interpretation of data and drafted the manuscript. YP and JW participated in the imaging analysis and carried out the acquisition and interpretation of data. YS and WL revised the manuscript critically for important intellectual content and gave final approval of the version to be published. All authors read and approved the final manuscript.

\section{Competing interests}

The authors declare that they have no competing interests.

\section{Disclosures}

This material has not been published and is not under consideration elsewhere. This study receives no financial support.

\section{Author details}

'Department of Orthopaedic Surgery, The Third Hospital of Hebei Medical University, 139 Ziqiang Road, Shijiazhuang 050051, People's Republic of China. ${ }^{2}$ The Key Laboratory of Orthopedic Biomechanics of Hebei Province, The Third Hospital of Hebei Medical University, Shijiazhuang 050051, People's Republic of China. ${ }^{3}$ Department of Orthopedic Surgery, Hebei General Hospital, 348 Heping Road, Shijiazhuang 050000, People's Republic of China. ${ }^{4}$ Department of ultrasound, Hebei General Hospital, 348 Heping Road, Shijiazhuang 050000, People's Republic of China.

Received: 6 January 2016 Accepted: 18 May 2016

Published online: 13 June 2016

\section{Reference}

1. Heck CA, Brown CR, Richardson WJ. Venous thromboembolism in spine surgery. J Am Acad Orthop Surg. 2008;16:656-64.

2. Nicol M, Sun Y, Craig N, Wardlaw D. Incidence of thromboembolic complications in lumbar spinal surgery in 1,111 patients. Eur Spine J. 2009;18:1548-52.

3. Shimoyama Y, Sawai T, Tatsumi S, Nakahira J, Oka M, Nakajima M, Jotoku T, Minami T. Perioperative risk factors for deep vein thrombosis after total hip arthroplasty or total knee arthroplasty. J Clin Anesth. 2012;24:531-6.

4. Oda T, Fuji T, Kato Y, Fujita S, Kanemitsu N. Deep venous thrombosis after posterior spinal surgery. Spine (Phila Pa 1976). 2000;25:2962-7.

5. Hansen MA, Fehlings MG. Preventing venous thromboembolism after thoracic or thoracolumbar spinal fusion. World Neurosurg. 2012;78:434-6.

6. Raj D, Marshall RW. Prophylaxis against thromboembolism in spinal surgery. Arch Orthop Trauma Surg. 2008;128:1365-71.

7. Strom RG, Frempong-Boadu AK. Low-molecular-weight heparin prophylaxis 24 to 36 hours after degenerative spine surgery: risk of hemorrhage and venous thromboembolism. Spine (Phila Pa 1976). 2013;38:E1498-502.

8. Hamidi S, Riazi M. Incidence of venous thromboembolic complications in instrumental spinal surgeries with preoperative chemoprophylaxis. J Korean Neurosurg Soc. 2015;57:114-8. 
9. Sudo A, Wada H, Nobori T, Yamada N, Ito M, Niimi R, Hasegawa M, Suzuki K, Uchida A. Cut-off values of D-dimer and soluble fibrin for prediction of deep vein thrombosis after orthopaedic surgery. Int J Hematol. 2009;89:572-6.

10. Jiang Y, Li J, Liu Y, Li YC, Zhang WG. Risk factors for deep vein thrombosis after orthopedic surgery and the diagnostic value of D-dimer. Ann Vasc Surg. 2015;29:675-81.

11. Glotzbecker MP, Bono CM, Wood KB, Harris MB. Postoperative spinal epidural hematoma: a systematic review. Spine (Phila Pa 1976). 2010;35: E413-20.

12. Smith JS, Fu KM, Polly Jr DW, Sansur CA, Berven SH, Broadstone PA, et al. Complication rates of three common spine procedures and rates of thromboembolism following spine surgery based on 108,419 procedures : a report from the Scoliosis Research Society Morbidity and Mortality Committee. Spine (Phila Pa 1976). 2010;35:2140-9.

13. Glotzbecker MP, Bono CM, Wood KB, Harris MB. Thromboembolic disease in spinal surgery: a systematic review. Spine (Phila Pa 1976). 2009;34:291-303.

14. Gerlach GR, Raabe A, Beck J, Woszczyk A, Seifert V. Postoperative nadroparin administration for prophylaxis of thromboembolic events is not associated with an increased risk of hemorrhage after spinal surgery. Eur Spine J. 2004; 13:9-13.

15. Sansone JM, del Rio AM, Anderson PA. The prevalence of and specific risk factors for venous thromboembolic disease following elective spine surgery. J Bone Joint Surg Am. 2010;92:304-13.

16. Yoshioka K, Murakami H, Demura S, Kato S, Hayashi H, Inoue K, Ota T, Shinmura K, Yokogawa N, Fang X, Tsuchiya H. Comparative study of the prevalence of venous thromboembolism after elective spinal surgery. Orthopedics. 2013;36:e223-8.

17. Yoshioka K, Kitajima I, Kabata T, Tani M, Kawahara N, Murakami H, Demura S, Tsubokawa T, Tomita K. Venous thromboembolism after spine surgery: changes of the fibrin monomer complex and D-dimer level during the perioperative period. J Neurosurg Spine. 2010;13:594-9.

18. Wakabayashi $H$, Hasegawa M, Niimi R, Sudo A. Clinical analysis of preoperative deep vein thrombosis risk factors in patients undergoing total hip arthroplasty. Thromb Res. 2015

19. Izumi M, Migita K, Nakamura M, Jiuchi Y, Sakai T, Yamaguchi T, Asahara T, Nishino Y, Bito S, Miyata S, Kumagai K, Osaki M, Mawatari M, Motokawa S. Risk of venous thromboembolism after total knee arthroplasty in patients with rheumatoid arthritis. J Rheumatol. 2015;42:928-34.

20. Tamura Y, Mori S, Asada S, Kawao N, Ueshima S, Kaji H, Yamamoto J, Akagi M, Matsuo O. Enhanced pre-operative thrombolytic status is associated with the incidence of deep venous thrombosis in patients undergoing total knee arthroplasty. Thromb J. 2014;12:11.

\section{Submit your next manuscript to BioMed Central and we will help you at every step:}

- We accept pre-submission inquiries

- Our selector tool helps you to find the most relevant journal

- We provide round the clock customer support

- Convenient online submission

- Thorough peer review

- Inclusion in PubMed and all major indexing services

- Maximum visibility for your research

Submit your manuscript at www.biomedcentral.com/submit

C Biomed Central 